\title{
scripted
}

Volume 15, Issue 1, August 2018

\section{Book review: Artificial Intelligence and Legal Analytics: New Tools for Law Practice in the Digital Age}

\author{
Kevin D. Ashley \\ Cambridge: Cambridge University Press, 2017. 426 pages. \\ ISBN 9781316622810. £34.99. \\ Reviewed by Jesus Manuel Niebla Zatarain* \\ (c) (1) $\odot$ \\ (C) 2018 Jesus Manuel Niebla Zatarain \\ Licensed under a Creative Commons Attribution-NonCommercial- \\ NoDerivatives 4.0 International (CC BY-NC-ND 4.0) license
}

DOI: $10.2966 /$ scrip.150118.156

* Professor and researcher, Faculty of Law, Autonomous University of Sinaloa, Mazatlán, Sinaloa, Mexico. Email: jmniebla@yahoo.com 
Can we fully represent cognitive legal processes through computational models? This is perhaps one of the most complex yet fascinating questions experts in the area of legal artificial intelligence are trying to solve. In this context, Kevin Ashley's Artificial Intelligence and Legal Analytics: New Tools for Law Practice in the Digital Age delivers a comprehensive study aimed at understanding the evolution of this interdisciplinary field. The author begins by presenting a description of early artificial intelligence developments implemented in the legal sphere which, regardless the fact they did not perform legal reasoning per se, led to the development of new applications.

An example of the latter are legal AI expert systems. These developments operate in a specific area of legal knowledge and construct a legal outcome through the direct interaction with its user. Nonetheless, these early devices had three primary downsides: first, they did not possess the capacity to represent uncertainty, which leads to incomplete legal data. Second, the process of providing the device with legal rules was time consuming and resource intensive, limiting their potential use. Third, this produced a bottleneck that could not be solved through text analytics. To increase the level of comprehension of this section of the text, further explanation along with additional illustration would have been convenient.

In relation to the previous point, the author clearly describes how researchers in $\mathrm{AI}$ and the law complemented the open texture approach implemented by early devices with computational models of legal reasoning (CMLR). This resulted in arguments that included legal text input, prediction of the problem's outcome, and an explanation in terms that were comprehensible by legal professionals with no technical background. Overall, this allowed legal AI to represent human legal knowledge in terms that can be used to develop relevant intelligent tools for the legal field. 
On a first look, it is established that the law itself is a domain of rules, susceptible to being presented through computational code interpretable through artificial reasoning. However, features such as vagueness and the opentexture of statutory provisions need to be addressed to create accurate legal reasoning. An approach that delivers this is the emulation of how courts provide their rulings. The process is decomposed into three parts: first, a theory based on previously existing cases that resemble the one currently being analysed is created. Second, these recently created patterns are used to strengthen or weaken an argument. Third, example-based explanations illustrate why an argument can or cannot be applied. It is within this scope that the first section of the book is presented.

The second section of the book addresses a technical aspect - the development of legal tools. Here the author proposes the use of ontologies to properly describe relevant relations within a specific area of the law. This is combined with machine learning techniques, which allows learning from the input received to maximise legal accuracy and operational efficiency. The result of this is evidence that supports the notion that argument-related data could be implemented to support conceptual legal information retrieval through computational platforms.

Here, the author makes a crucial contribution: if models of legal reasoning and argumentation are to have a greater impact on legal practice, they will likely need to do so by including pre-existing commercial and institutional approaches to full-text legal information retrieval and e-discovery (p. 210). The cooperation between legal AI and pre-existing approaches allows the management and processing of case decisions, statutes, and other relevant legal documents, providing tools that may be beneficial for practitioners and students.

In relation to legal documents as source of legal knowledge the importance of machine learning techniques is highlighted by the author. Here, 
the device detects relevant patterns to find correlations in new inputs. By this mechanism correlations between the instant case and similar precedents can be established. This by itself is a significant advance: unlike traditional predictive methods that were designed to operate only on Supreme Court decisions, this new approach is capable to represent features obtained from private litigation.

This technical approach supports the development of cognitive computing, which functions not by solving the problem presented by one of the plaintiffs but by providing new forms of relevant information that can assist in solving a specific situation. To apply machine learning techniques to legal texts a threestep method is implemented: first, collecting and processing raw data. Second, transforming the raw data into a uniform linguistic element. Third, delivering the document as a feature vector that recognizes particular features in the legal text.

Regardless of the apparent benefits of this approach, at the time of the book's publication, there had not yet been any developments that fully implement the method described. One of the reasons for this is the actual design limitation that legally relevant devices face. To illustrate this, the author discusses two systems, Watson and Debater. The first operates on corpus of text, gathering relevant information that it uses to provide an answer for the question it receives. A downside of this approach is that it only operates on the text provided, which is likely to be too narrow for complex implementations. Additionally, in law it is crucial to provide an argument (the logical description of the reasoning process) that led to an outcome, something that is also not provided by this system. In relation to Debater, it is presented as an improved version of Watson, capable of constructing relevant legal arguments. Apart from this, the author also proposes further adaptation of Watson's operative aspects in order to enhance its operation when applied to the legal field. 
At this point, the author establishes that understanding regulatory texts is a fundamental requirement to properly apply the legal rules provided by the legislator. This makes the insertion of legal AI a natural step towards the extraction and correct interpretation of relevant legal data in a large textual corpus. In this scenario, the implementation of different technological methods to obtain relevant data and the use of rule-based approaches to classify statutory provisions from relevant sources are proposed. In this sense, chapter 9 addresses the extraction of logical rules from statements and regulations by implementing deductive and defeasible approaches. Here, the author uses environmental designs to deliver a view of how these techniques operate (through a compliant design).

The third and last section of this book presents the LUIMA (Legal Unstructured Information Management Architecture) design, evaluating its composition and the contribution it makes to any full text legal information system. The outcome provided by this platform outperforms those developed under traditional approaches, since it addresses semantic elements contained in legal documents. This means that the system can distinguish between sentences that represent a rule and sentences that state findings of fact.

Finally, the author recognizes that regardless the current advances and the on-going effort to extract rules from regulatory texts, they are still far from ideal. Nonetheless, the advances obtained are being used in other applications, such as construction of legal abstracts or statutory provisions and the relation between relevant agents according to the nature of the data contained in these texts. A positive aspect of this work is that it properly illustrates the suitability of emulating human cognitive processes and their use in the development of functional legal technology.

In relation to the composition of this book, it provides a comprehensive and user-friendly description of this interdisciplinary area, focusing on the 
suitability of developing legal devices based on artificial intelligence. The structure of the work allows users to analyse how representation of legal logic knowledge occurs, and its suitability for computational implementations (pp. 173-175). On this matter, the author provides relevant and understandable illustrations that facilitate the linkage between theory and the development of the techno legal implementations. In this aspect, is worth mentioning the form in which relevant technological approaches, such as machine learning and the extraction of argument related information, were addressed. The author also makes the transition to current technology in a continuous and reader-friendly form, highlighting current methods of information retrieval that increase the quality of the legal outcome provided. However, some recommendations can be proposed to this work. To increase the clarity of the illustrations provided, further explanation is suggested, especially for audiences that lack a computer science background.

In summary, Artificial Intelligence and Legal Analytics: New Tools for Law Practice in the Digital Age is a fundamental work for those of us who are interested in the intersection between intelligent technology and the legal field, and its promising future. 